\title{
Impact dynamics of micromachined bidirectional electrothermal vibromotors
}

\author{
Wen-Pin Shih \\ Department of Mechanical Engineering, National Taiwan University, Taiwan \\ Minfan Pai \\ Discera, Inc., Ann Arbor, Michigan 48105 \\ Yu-Yun Lin \\ Department of Civil Engineering, National Cheng-Kung University, Taiwan \\ Chung-Yuen Hui \\ Department of Theoretical and Applied Mechanics, Cornell University, Ithaca, New York 14853 \\ Norman C. Tien \\ Berkeley Sensor and Actuator Center, Department of Electrical and Computer Engineering, University of \\ California, Davis, California 95616
}

(Received 9 April 2004; accepted 18 August 2004)

\begin{abstract}
The motion of micromachined bidirectional electrothermal vibromotors (BETV) is analyzed using an impact model that takes into account friction and contact, as well as the compliance of the slider and the impact head. This model addresses several important issues on the usage of BETV. These include (a) how to achieve the pull and push mode; (b) avoiding multiple impacts in one actuation cycle; (c) the existence of periodic solutions with the same period as the thermal actuator. Four dimensionless parameters are found to govern the dynamics of the vibromotor. Depending on these parameters, we observed periodic motion, period doubling, and aperiodic solution. A criterion is established to separate push and pull modes. An experiment that includes fabricating and characterizing a BETV is carried out to verify the ability of the model to predict the pull mode.

(c) 2004 American Institute of Physics. [DOI: 10.1063/1.1805725]
\end{abstract}

\section{INTRODUCTION}

Micromachined linear vibromotors are commonly used to move microscale components for long distances. They have been applied to optical communication systems and self-assembly technology. ${ }^{1-5}$ These vibromotors are driven either electrostatically or electrothermally. Generally, electrothermal vibromotors have a smaller footprint and require lower driving voltage than electrostatic vibromotors. The scanning electron microscope (SEM) image of a typical electrothermal vibromotor (ETV) fabricated by standard silicon surface micromachining is shown in Fig. 1. The slider is composed of two parallel beams connected at both ends. It is driven by actuators symmetrically placed at an angle $\theta$ on both sides of the slider. A typical electrothermal actuator has two conductive arms of differing widths through which electric current flows. ${ }^{6-9}$ The cold arm consists of an impact and a flexure element. The width of the impact element is much greater than that of the hot arm, resulting in a much larger current density in the latter. Ohmic heating of this arm causes it to expand more than the cold arm. This causes the actuator to deflect and impact the slider. Opposing sets of actuators allow bidirectional motion to be achieved.

The development of the vibromotor has been limited by incomplete understanding of its impact mechanics. For example, even if the actuators are driven periodically, the motion of the slider need not be periodic. Furthermore, multiple impacts can occur during one actuation cycle. Experiments have shown that in many instances the slider actually moves opposite to the impact direction, resulting in the "pull mode." ${ }^{10}$ In addition, the slider can alternate between pull and push modes resulting in the negligible displacement. ${ }^{11}$ Specifically, if the net (positive) displacement of the slider is in the impact direction after one cycle of actuation, the slider is said to be in push mode during that cycle. On the other hand, the slider is in pull mode during a cycle if it has negative (opposite to the impact direction) displacement after an actuation cycle. The pull mode is desirable in some applications. The dynamics of the micromachined vibromotors have been studied using a nonzero coefficient of restitution by Lee, Pisano, and Lin and Danemon et al. ${ }^{12,13}$ The impact process is assumed to be instantaneous and the impact force is impulsive. A drawback of this model is that it cannot pre-

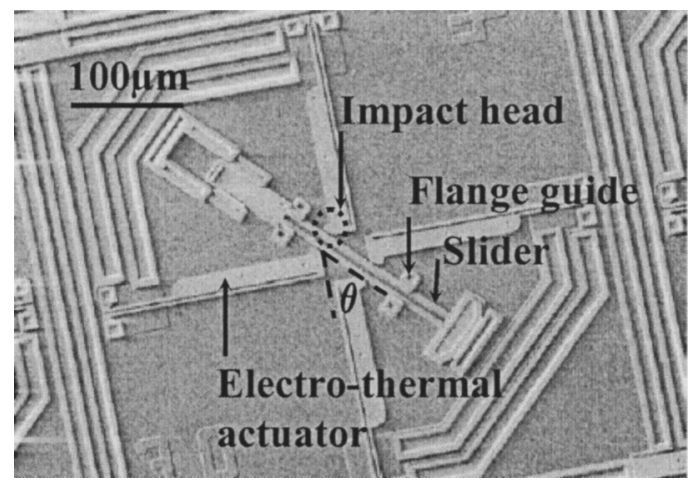

FIG. 1. SEM image of micromachined electrothermal vibromotor 


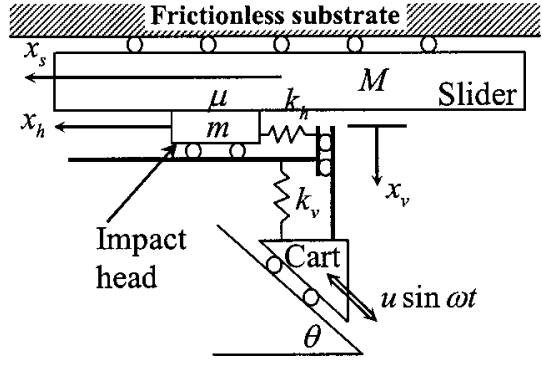

FIG. 2. Impact mechanics model.

dict pull mode. To overcome these shortcomings, we consider a continuous impact model which allows the slider and the actuator to interact in a continuous manner during contact. A complete literature survey of various impact model can be found in the work of Gilardi and Sharf. ${ }^{14}$ Based on this model, we studied the existence and dynamics of push and pull modes and investigated the possibility of periodic motion. We also studied the problem of multiple impacts. A parameter study based on this model was carried out from the viewpoint of device design and operation. Some aspect of our theory was compared with experimental results.

\section{IMPACT MODEL}

Figure 2 depicts the impact model in this work. The parameters used in this model are summarized in Table I. One half of the vibromotor is modeled due to the symmetry of the device. The rollers between the slider and the wall represent frictionless contact between the slider and the substrate. The impact head, which is attached to the impact element (Fig. 1), is represented by a pad of mass $m$. Impact results from the collision of the pad to the slider. We assume that there is no adhesion between the impact head and the slider and the impact is plastic, i.e., the coefficient of restitution is zero in the vertical direction. Impact compliance in the horizontal and vertical directions is modeled by two linear springs of stiffness $k_{h}$ and $k_{\nu}$, respectively. The cart, which represents the electrothermal actuator, is constrained to move on a plane inclined at an angle $\theta$ (impact angle) with the slider (Fig. 1). Cart motion is assumed to be periodic; that is, the displacement of the cart is assumed to be $u \sin \omega t$,

TABLE I. Model parametes. The symbols inside brackets are normalized parameters.

\begin{tabular}{cc}
\hline \hline Parameter & Intepretation \\
\hline$M$ & One half of slider mass \\
$m$ & Mass of impact head \\
$k_{h}$ & Horizontal impact compliance \\
$k_{v}$ & Vertical impact compliance \\
$\theta$ & Impact angle \\
$u$ & Maximum deflection of actuator \\
$\omega$ & Angular frequency of vibration \\
$t$ & Time \\
$x_{h}$ & Horizontal displacement of impact head \\
$x_{v}$ & Vertical displacement of impact head \\
$x_{s}$ & Slider displacement \\
$\mu$ & Coulomb friction coefficient \\
\hline \hline
\end{tabular}

where $u$ is the maximum deflection of the actuator and $\omega$ is the angular frequency of the vibration. This assumption implies that the motion of the thermal actuator is unaffected by impact. Let $\mathbf{X}$ be a coordinate system fixed to the side of the slider. Let the vertical displacement of the pad be denoted by $\mathbf{X}=x_{\nu}$ so that the impact occurs when $x_{\nu}=0$. Note $x_{\nu} \geqslant 0$ since the pad cannot penetrate the slider. Without loss of generality, we assume first impact occurs at $t=0$, when the vertical position of the cart with respect to the coordinate system $\mathbf{X}$ is zero $\left(x_{\nu}=0\right)$ and the vertical and horizontal springs are both relaxed. For $x_{\nu}>0$ (i.e., when the impact head swings backward), there is no contact between the slider and the impact head, so that the force acting on the slider is zero. Note that the tension $T$ in the vertical spring is given by $T=-k_{\nu}\left(u \sin \theta \sin \omega t+x_{\nu}\right)$. The absence of adhesion implies that contact is impossible if $T>0$. For the rest of this paper, $T>0$ means that there is no contact even though $x_{\nu}$ can be zero; that is, $x_{\nu}=0$ is a necessary condition for contact. Note that, since $x_{\nu}=0$ during contact, $T$ $=-k_{\nu} u \sin \theta \sin \omega t$, this means that $T>0$ only if $\sin \omega t<0$. As a result, detachment of the impact head from the slider can be determined by checking whether the condition $\sin \omega t<0$ is satisfied. Note that the condition $\sin \omega t<0$ does not always imply $x_{\nu}>0$, since the impact head can touch the slider while the vertical spring is in tension.

Impact friction is assumed to follow Coulomb's law, i.e.,

$$
F=\mu N \operatorname{sgn}\left(\nu_{R}\right)
$$

where $\mu$ is the friction coefficient, $F$ is the force on the slider, and $N$ is the normal contact force. $\nu_{R}$ is velocity of impact head relative to the slider, i.e.,

$$
\nu_{R}=\dot{x}_{h}-\dot{x}_{s},
$$

where the horizontal displacements of the impact head and slider are denoted by $x_{h}$ and $x_{s}$, respectively; $\dot{x}_{h}$ and $\dot{x}_{s}$ denote the differentiation with respect to time. The sgn function is defined by

$$
\begin{aligned}
& \operatorname{sgn}\left(\nu_{R}\right)=1, \quad \nu_{R}>0(\operatorname{slip}), \\
& \operatorname{sgn}\left(\nu_{R}\right)=-1, \quad \nu_{R}<0 \text { (reverse slip), } \\
& \left|\operatorname{sgn}\left(\nu_{R}\right)\right| \leqslant 1, \quad \nu_{R}=0 . \quad \text { (stick) }
\end{aligned}
$$

For $x_{\nu}>0$ or $x_{\nu}=0$ and $\sin \omega t<0$, the slider and the impact head are not in contact. In this case, the equations of motion are

$$
\begin{aligned}
& m \ddot{x}_{\nu}=-k_{\nu}\left(u \sin \theta \sin \omega t+x_{\nu}\right), \\
& m \ddot{x}_{h}=k_{h}\left(u \cos \theta \sin \omega t-x_{h}\right), \\
& \ddot{x}_{s}=0,
\end{aligned}
$$

where $x_{\nu}$ denotes the vertical displacement of the pad.

During contact, the vertical spring is in compression $(T<0)$ and $x_{\nu}=0$ so that $\sin \omega t \geqslant 0$. Since the collision is assumed to be plastic, $x_{\nu}=0$ and $\dot{x}_{\nu}=\ddot{x}_{\nu}=0$ immediately after impact. The equations of motion during contact are 


$$
\begin{aligned}
m \ddot{x}_{h}= & k_{h}\left(u \cos \theta \sin \omega t-x_{h}\right) \\
& -\mu k_{\nu} u \sin \theta \sin \omega t \operatorname{sgn}\left(\dot{x}_{h}-\dot{x}_{s}\right), \\
M \ddot{x}_{s}= & \mu k_{\nu} u \sin \theta \sin \omega t \operatorname{sgn}\left(\dot{x}_{h}-\dot{x}_{s}\right),
\end{aligned}
$$

where $k_{\nu} u \sin \theta \sin \omega t$ is the normal compressive force on the slider, and $M$ is the half mass of the slider.

To reduce the number of parameters, these equations of motion are normalized using the dimensionless variables: $\xi \equiv x_{\nu} / u \sin \theta, \quad \zeta \equiv x_{h} / u \cos \theta, \quad s \equiv x_{s} / u \cos \theta$, $\tau \equiv t \sqrt{k_{\nu} / m}$. Equations (2a)-(2e) in normalized form are Noncontact phase. $\xi>0$ or $\xi=0$ and $\sin \varpi \tau<0$ :

$$
\begin{aligned}
& \xi^{\prime \prime}+\xi=-\sin \varpi \tau, \\
& \alpha \zeta^{\prime \prime}+\zeta=\sin \varpi \tau, \\
& s^{\prime \prime}=0 .
\end{aligned}
$$

Contact phase. $\xi=0$ and $\sin \varpi \tau \geqslant 0$ :

$$
\begin{aligned}
& \xi=0, \\
& \alpha \zeta^{\prime \prime}+\zeta=\left[1-\alpha \kappa \operatorname{sgn}\left(\zeta^{\prime}-s^{\prime}\right)\right] \sin \varpi \tau, \\
& s^{\prime \prime}=\beta \kappa \operatorname{sgn}\left(\zeta^{\prime}-s^{\prime}\right) \sin \varpi \tau,
\end{aligned}
$$

where $\varpi \equiv \omega \sqrt{m / k_{\nu}}, \alpha \equiv k_{\nu} / k_{h}, \beta \equiv m / M, \kappa \equiv \mu \tan \theta$, and $\xi^{\prime} \equiv d \xi / d \tau$. Based on the aforementioned assumptions of initial conditions, the initial displacement and velocity and velocity for the slider are zero at $\tau=0$. The initial displacement and velocity of the impact head are zero (i.e., contact is about to occur) in both vertical and horizontal directions.

\section{VERTICAL MOTION OF IMPACT HEAD}

Equations (3a)-(3f) imply that vertical motion of the impact head is decoupled from the horizontal motion. Specifically, according to Eqs. (3a) and (3d), the vertical motion of the impact head depends only on the normalized angular frequency $\varpi$ of the actuation and is not affected by the friction interaction with the slider. In this section we address the vertical motion of the impact head, which is governed by Eq. (3a) during the noncontact phase and by Eq. (3d) during the contact phase, where the force in the vertical spring is compressive. Note that the vertical spring in the noncontact phase can either be in tension or in compression. This characteristic of the vertical motion is similar to a linear impact oscillator studied by Shaw and Holmes. ${ }^{15}$ It should be noted that although the impact head is driven periodically with the actuation frequency $\varpi$, the motion of the impact head need not be periodic. Furthermore, even if the motion were periodic, the impact frequency is not necessarily the same as the actuation frequency $\varpi$, as shown below. Physically, this translates to multiple impacts in one actuation cycle.

To fix ideas, consider a general impact cycle shown in Fig. 3. An impact period starts at $\tau=\tau_{1}$ when the impact head hits the slider with negative velocity and the vertical spring in compression (point $\mathbf{0}$ ). Since $\sin \varpi \tau>0$ is necessary for the vertical spring in compression, $2 m \pi / \varpi<\tau_{1}<(2 m$ $+1) \pi / \varpi$, where $m$ is nonnegative integer. The plastic impact

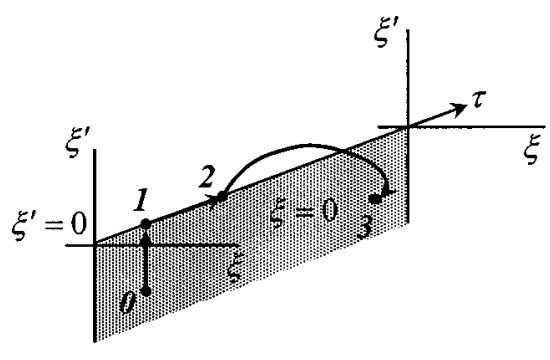

FIG. 3. Phase space $\left(\tau, \xi, \xi^{\prime}\right)$ showing trajectory of one impact period of the impact head.

assumption implies that the vertical velocity of the impact head becomes zero right after the impact (the point $\mathbf{0}$ is mapped to 1). The impact head remains in contact with the slider until the vertical spring becomes tensile. Separation starts at $\sin \varpi \tau_{2}=0$ (point 2), where $\tau_{2}=(2 m+1) \pi \geqslant \tau_{1}$. When the cart moves backward at $\tau_{2}$, the vertical spring is relaxed from compression and will be stretched for $\tau>\tau_{2}$. As a result, $\tau_{2}-\tau_{1}<\pi / \varpi$. Once contact is broken, the motion of the impact head is determined by the solution of Eq. (3a) and has the general form,

$$
\xi=a \sin \tau+b \cos \tau+\left(\varpi^{2}-1\right)^{-1} \sin \varpi \tau, \quad \tau \geqslant \tau_{2},
$$

where we have assumed that $\varpi \neq 1$. The integration constants, $a$ and $b$, are determined by the condition $\xi\left(\tau_{2}\right)$ $=\xi^{\prime}\left(\tau_{2}\right)=0$. This results in

$$
\xi=\left(\varpi^{2}-1\right)^{-1}\left[\varpi \sin \left(\tau_{2}-\tau\right)-\sin \varpi \tau\right], \quad \tau>\tau_{2} .
$$

Note that $\xi$ given by Eq. (4b) is positive until $\tau=\tau_{3}$, where $\tau_{3}$ is the first root of the equation,

$$
\sin \varpi \tau=\varpi \sin \left(\tau_{2}-\tau\right),
$$

which is greater than $\tau_{2}$. At $\tau=\tau_{3}$, the impact head hits the slider again (point 3 in Fig. 3). Note that, depending on $\varpi, \tau_{3}$ can be smaller than $\tau_{2}+\pi / \varpi$. That is, depending on $\varpi$, it is possible that multiple impacts can occur before $\tau$ researches $\tau_{1}+2 \pi / \varpi$, i.e., before the next actuation cycle occurs.

The above analytic method is simply too tedious to carry out for multiple impacts. Therefore, we studied the motion by numerically integrating Eqs. (3a) and (3d) for 30 consecutive impacts with the initial conditions $\xi(0)=\xi^{\prime}(0)=0$. Calculations are also performed with 100 consecutive impacts. The results of these calculations are identical to those obtained using 30 impacts. Let $P$ denote the time between two consecutive impacts. The normalized duration between impacts $\hat{P}=P \varpi / 2 \pi$ or impact period is shown in Fig. 4 for $0<\varpi \leqslant 10$. Note that $0<\varpi \leqslant 10$ corresponds to the real range of resonance frequencies of typical electrothermal actuators. A normalized period of 1 indicates that the solution is periodic and the impact period is identical to the actuation period of the thermal actuator. Figure 4(a) shows that, for $0<\varpi<1 / 2$, there are regions where two impacts occur during one actuation cycle. One of these impacts occurs at a higher frequency than the actuation frequency of the thermal actuator while the second impact occurs at about the same frequency. We define this as period doubling since the impact head hits the slider with two different frequencies. All the solutions in Fig. 4(a) are periodic. Figure 4(b) shows that, 


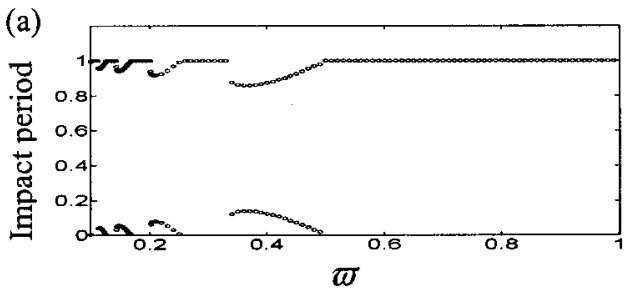

(b)

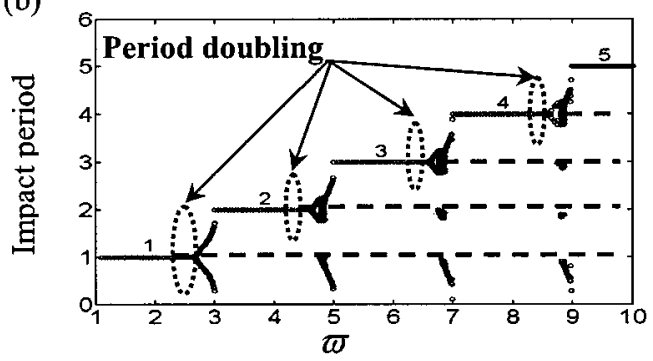

FIG. 4. Bifurcation diagram summarizing the periodic motion of the impact head in the vertical direction: (a) $0<\varpi<1$, (b) $1 \leqslant \varpi<10$. Dots are numerical data. Number above branch indicates the impact period, which is the impact duration normalized with the actuation period of the thermal actuator $(2 \pi / \varpi)$. The regions where period doubling occurs are enclosed by dotted ellipses.

for $\varpi>2$, periodic solution of Eqs. (3a) and (3d) exists with impact period being integer multiples of the actuation period $2 \pi / \varpi$. For example, for $3<\varpi \leqslant 4$, the impact period is 2 , so that one impact occurs for every two actuation cycles. Figure 4(b) also shows that period doubling occurs at $\varpi \sim 2 n$ +0.64 , where $n$ is the ratio of the impact period to the actuation period $2 \pi / \varpi$ just before the occurrence of period doubling. Except for $0<\varpi<1 / 2$, the regions where period doubling occurs are enclosed by dotted ellipses in Fig. 4(b). For $\varpi>4.64$ or $n>2$, there exist periodic regions $2 n$ $-1<\varpi<2 n+0.64$. These regions are followed by period doubling region which in turn are followed by aperiodic regions, where periodic solution of Eqs. (3a) and (3d) cannot be found [see also Fig. 5(c)]. For example, $\varpi=4.65$ is in the period doubling region and $\varpi=4.8$ is in aperiodic region. The impact periods in aperiodic regions occur in a random way and do not follow simple patterns.

The trajectories of the periodic motion of the impact head are shown in Figs. 5(a) and 5(b) for different values of $\varpi$. Note that the trajectories in these figures are discontinuous at $\xi=0$. The trajectories during the noncontact phase $(\xi>0)$ are shown by smooth curves. During the contact phase $(\xi=0), \xi^{\prime}$ is mapped from negative value to zero due to plastic impact. Figure 5(c) shows the trajectories of ten consecutive impact of the aperiodic solution when $\varpi=4.8$.

The numerical results in Fig. 4 indicate that the impact period is identical to the actuation period of the thermal actuator only when $0.5 \leqslant \varpi \leqslant 2$.6. In addition, the impact period is always a multiple of actuation period when $\varpi$ is outside of the period doubling and aperiodic regions. Since the motion of the impact head in these period doubling and aperiodic regions is complex or unpredictable, we restrict our analysis in the frequency range $0.5 \leqslant \varpi \leqslant 2$. When $\varpi=1$, the impact head vibrates in the vertical direction with a frequency close to the resonance frequency of typical electrothermal actuators. It should also be noted that even if the
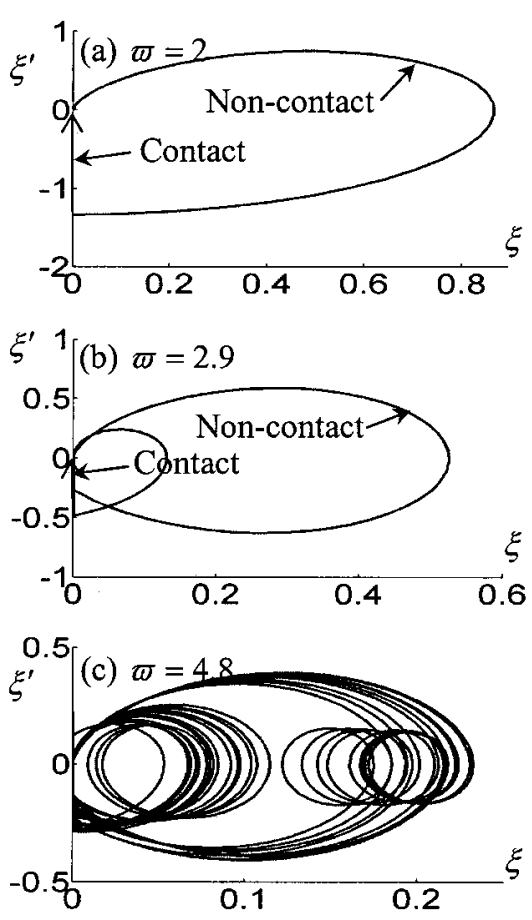

FIG. 5. Trajectories of the vertical motion of the impact head under different actuation frequencies of the thermal actuator. (a) Periodic solution, $\varpi=2$; (b) period doubling, $\varpi=2.9$; (c) aperiodic solution, $\varpi=4.8$.

actuation frequency is in the range where periodic impact exists, i.e., $0.5 \leqslant \varpi \leqslant 2.6$, periodic motion of the slider is not assured. Two issues come into play as far as the periodicity of the slider is concerned. The first is the interaction of the slider with the impact head in the horizontal direction, which will be studied next. The second is the contact duration. The numerical results in Fig. 6 show that the contact duration in an impact period vanishes for $2 \leqslant \varpi<2$.6. Physically, this means the impact head leaves the slider immediately after impact because the vertical spring is under tension. In this case, the motion of the slider is governed by an impulse force and cannot be predicted by our continuous contact model. This phenomenon can be eliminated using an adhesion model for the contact interfaces. Since adhesion is not considered in this work, only actuation frequencies in the range $0.5 \leqslant \varpi<2$ will be considered below.

\section{SLIDER DYNAMICS FOR $0.5 \leqslant \varpi<2$}

The preceding section shows that the vertical motion of the impact head is periodic as long as the actuation frequen-

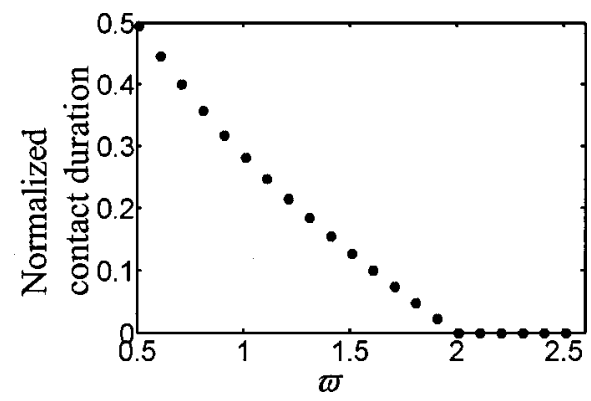

FIG. 6. Contact duration vs angular actuation frequency. Here the contact duration is normalized by actuation period $(2 \pi / \varpi)$. 
cies of the thermal actuator are confined within $0.5 \leqslant \varpi<2$. In this range, the normalized impact period is $2 \pi / \varpi$ and impulsive impact does not exist. When the vertical motion reaches its periodic state, the impact head separates from the slider at $\tau_{2}=(2 n-1) \pi$, where $n$ is a positive integer, since $\sin \varpi \tau_{2}=0$. Substituting $\tau_{2}=(2 n-1) \pi$ into Eq. (4c), the next contact occurs at $\tau_{3}$, where $\varpi \sin \tau_{3}=\sin \varpi \tau_{3}$ and $\tau_{3}>\tau_{2}$. In this section, the vertical motion of the impact head is assumed to be in periodic state (i.e., $0.5 \leqslant \varpi<2$ ) and the conditions which favor the push or pull modes are studied. Periodic motion of the slider is also investigated.

The dynamics of the push/pull mode and the conditions for periodic motion are governed by the interplay between slip, stick, and reverse slip when the slider and the impact head are in contact. Specifically, slip occurs when the impact head has greater horizontal velocity than the slider, i.e., $\zeta^{\prime}>s^{\prime}$. Conversely, reverse slip is defined by $\zeta^{\prime}<s^{\prime}$. When the slider and the impact head reach the same velocity during contact, stick $\left(\zeta^{\prime}=s^{\prime}\right)$ will occur provided that the condition $\zeta^{\prime \prime}=s^{\prime \prime}$ is also satisfied.

Suppose the slider and the impact head both have positive velocity in the beginning of an impact cycle and $\zeta^{\prime}>s^{\prime}$. Friction force will impede the motion of the impact head while increases the velocity of the slider. If the contact condition changes from slip to reverse slip during impact, the slider could maintain positive velocity when the impact head separates from the slider. In this case, the slider will have a net positive displacement at the end of the impact cycle and is hence in push mode. On the other hand, the slider and the impact head would move together with identical negative velocity if the contact changes from slip to stick. This could cause pull mode, i.e., negative net displacement to the slider. The condition $\zeta^{\prime \prime}=s^{\prime \prime}$, together with Eqs. (1e), (3e), and (3f) implies that stick occurs if

$$
[1-\alpha \kappa(1+\beta)] \sin \varpi \tau \leqslant \zeta \leqslant[1+\alpha \kappa(1+\beta)] \sin \varpi \tau .
$$

Similarly, slip or reverse slip will occur when $\zeta^{\prime}=s^{\prime}$ if $\zeta^{\prime \prime}>s^{\prime \prime}$ or $\zeta^{\prime \prime}<s^{\prime \prime}$, respectively. These conditions correspond to

$$
\begin{aligned}
& \zeta<[1-\alpha \kappa(1+\beta)] \sin \varpi \tau \text { (slip), } \\
& \zeta>[1+\alpha \kappa(1+\beta)] \sin \varpi \tau \text { (reserve slip). }
\end{aligned}
$$

Based on Eqs. (5a)-(5c), the equations of motion in (3e) and (3f) can be simplified to piecewise linear differential equations, which are

Slip. $\zeta^{\prime}>s^{\prime}$ or $\zeta^{\prime}=s^{\prime}$ and Eq. (5b) is satisfied:

$$
\begin{aligned}
& \alpha \zeta^{\prime \prime}+\zeta=(1-\alpha \kappa) \sin \varpi \tau, \\
& s^{\prime \prime}=\beta \kappa \sin \varpi \tau .
\end{aligned}
$$

Reverse slip. $\zeta^{\prime}<s^{\prime}$ or $\zeta^{\prime}=s^{\prime}$ and Eq. (5c) is satisfied:

$$
\begin{aligned}
& \alpha \zeta^{\prime \prime}+\zeta=(1+\alpha \kappa) \sin \varpi \tau, \\
& s^{\prime \prime}=-\beta \kappa \sin \varpi \tau .
\end{aligned}
$$

Stick. $\zeta^{\prime}=s^{\prime}$ and Eq. (5a) is satisfied:
TABLE II. Governing parameters.

\begin{tabular}{ccc}
\hline \hline Parameter & Definition & Interpretation \\
\hline$\alpha$ & $k_{v} / k_{h}$ & Relative impact compliance \\
$\beta$ & $m / M$ & Relative mass \\
$\kappa$ & $\mu \tan \theta$ & Normalized friction force \\
$\varpi$ & $\omega \sqrt{m / k_{v}}$ & Normalized vibration frequency \\
\hline \hline
\end{tabular}

$$
\alpha(1+1 / \beta) \zeta^{\prime \prime}+\zeta=\sin \varpi \tau .
$$

The behavior of the solution depends on four parameters $(\alpha, \beta, \kappa, \varpi)$. The definitions of these parameters are summarized in Table II. To evaluate the effects of these four parameters on the dynamics of the slider, Eqs. (6)-(8) are numerically integrated assuming vertical motion of the impact head is periodic (i.e., $0.5 \leqslant \varpi<2$ ). The initial conditions $s^{\prime}(0)$ $=s(0)=\zeta^{\prime}(0)=\zeta(0)=0$ are used. Each simulation is carried out with fixed values of $\alpha, \beta$, and $\kappa$. A total of 720 simulations are carried out. The values of $\alpha, \beta$, and $\kappa$ used in these simulations are $\quad \kappa=0.1,0.4 ; \quad \beta=0.1,0.5,1,1.5 ; \quad \alpha$ $=0.1,0.5,1,1.5,2,2.5,3,3.5,4$. $\quad$ ๘ is swept from 0.5 to 2 with increment of 0.1 . Numerically, the slider motion is said to be periodic if the net displacement between two actuation cycles differs by less than $10^{-4}$. Unlike the vertical motion, where periodic motion is achieved within 30 impact cycles, the number of impact cycles required to achieve periodic slider motion depends on the values of the parameters and is sensitive to the initial conditions. The number of cycles required to reach periodic state varies from 300 to $10^{5}$ cycles. To ensure convergence of periodic solutions, we also monitor Poincare's section $\tau=2 m \pi / \varpi$ of the phase space $\left(\Delta s_{m}, s^{\prime}, \zeta, \zeta^{\prime}, \tau\right)$, where

$$
\Delta s_{m}=s[\tau=2(m+1) \pi / \varpi]-s(\tau=2 m \pi / \varpi), m \geqslant 1
$$

is the displacement increment in $m$ th actuation cycle.

Figure 7 shows four examples of our integration results for $(\kappa, \beta)=(0.1,0.1),(0.1,1.5),(0.4,0.1)$, and $(0.4,1.5)$, respectively. Push mode, shown as dots in Fig. 7, is obtained if
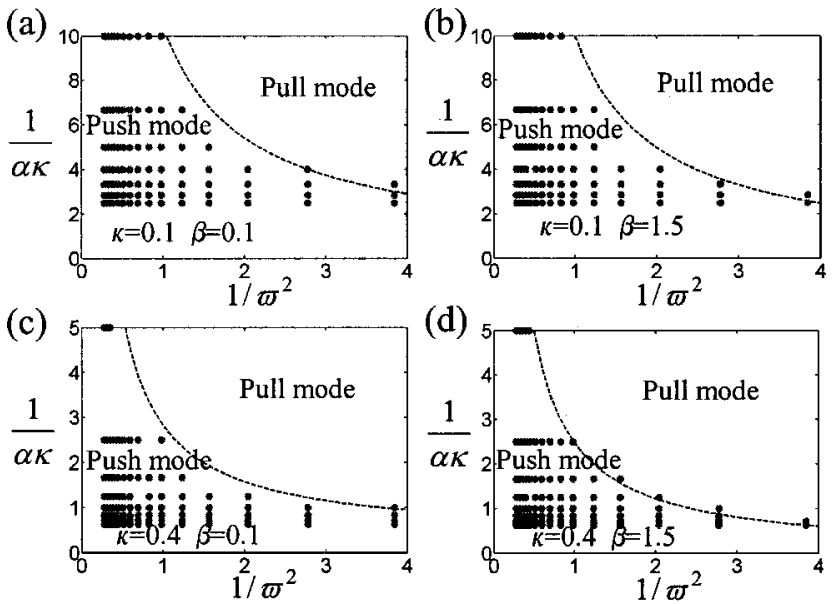

FIG. 7. Integration results of push and pull modes when the slider is in periodic state. Push mode is denoted by dots. The separation between the push and pull modes is represented by a dashed line. Different parameters of $\kappa$ and $\beta$ are used for the numerical integration: (a) $\kappa=0.1, \beta=0.1$; (b) $\kappa$ $=0.1, \beta=1.5$; (c) $\kappa=0.4, \beta=0.1$; (d) $\kappa=0.4, \beta=1.5$. 
the displacement increment in one impact cycle $\Delta s$ is positive. Pull mode is obtained if the displacement increment is negative. It should be noted that periodic solution exists for all combinations of $(\alpha, \beta, \kappa, \varpi)$. Therefore, the push and pull modes in Fig. 7 are evaluated based on the displacement increment in one period. Our numerical results show the following important result: there exists a curve with equation

$$
\frac{1}{\varpi^{2}}\left[\frac{1}{\alpha \kappa}-\frac{-\beta+1 / \beta}{22(\kappa+1)}\right]=\frac{1}{\kappa}
$$

in the $\left(1 / \varpi^{2}, 1 / \alpha \kappa\right)$ plane such that all push modes lie to the left of this curve whereas all pull modes lie to the right. The manner by which Eq. (9) is found will be explained below. Our numerical results also show that increasing $\varpi$ and $\alpha$ will provide a larger operation window for the push mode. This is because increasing the actuation frequency, $\varpi$, decreases the stick duration. Decreasing the stick duration will result in smaller negative slider displacements which favor the push mode. Likewise, decreasing the actuation frequency tends to suppress the push mode.

An important insight can be gained by studying the case $\alpha=k_{\nu} / k_{h} \rightarrow 0$, where the horizontal impact compliance vanishes. The absence of the horizontal spring implies that the impact head has the same motion as the impact element (the cart in Fig. 2) and hence multiple horizontal impacts within one actuation cycle cannot occur. Indeed, substituting $\alpha=0$ into Eqs. (3b) and (3e) the horizontal motion of the impact head both in contact and noncontact phases is

$$
\zeta=\sin \varpi \tau .
$$

Numerically, we found that the slider motion will always be driven into a periodic motion, irrespective of the initial conditions, as long as the vertical motion of the impact head is periodic (i.e., $0.5 \leqslant \varpi<2$ ). This condition will be assumed in the discussion below. Substitute $\alpha=0$ in Eqs. (3e) and (5a), it is easy to show that (5a) is satisfied so that stick will always occur whenever the slider and the impact head reach the same velocity during contact. In addition, the slider velocity during stick contact is

$$
s^{\prime}=\varpi \cos \varpi \tau .
$$

When the slider sticks to the impact head, both the governing equations, (5a) and (8), are satisfied until separation occurs when $\sin \varpi \tau=0$, i.e., $\tau=(2 n-1) \pi / \varpi$. Using Eq. (10b), the velocity of the slider at the instant of separation is $s^{\prime}=-\varpi$. If $s^{\prime}=-\varpi$ is used as the initial condition at $\tau=(2 n-1) \pi / \varpi$, then it can be directly verified that the slider motion is periodic. Thus, this implies that if stick occurs, then a periodic motion will result after separation. To understand our numerical results which show that the slider motion will always be driven into periodic motion, we demonstrate that all initial conditions will eventually lead to sticking of the slider to the impact head. Consider two scenarios in Fig. 8, where $s^{\prime} / \varpi$ is plotted against the normalized time $\varpi \tau / \pi$. In Fig. 8, the dotted sinusoidal curve is Eq. (10b) and is the velocity of the impact head. The first scenario corresponds to the slider starting with a velocity higher than the impact head at $\varpi \tau / \pi=1$, when separation has just occurred. After separation, the slider moves with constant velocity until contact

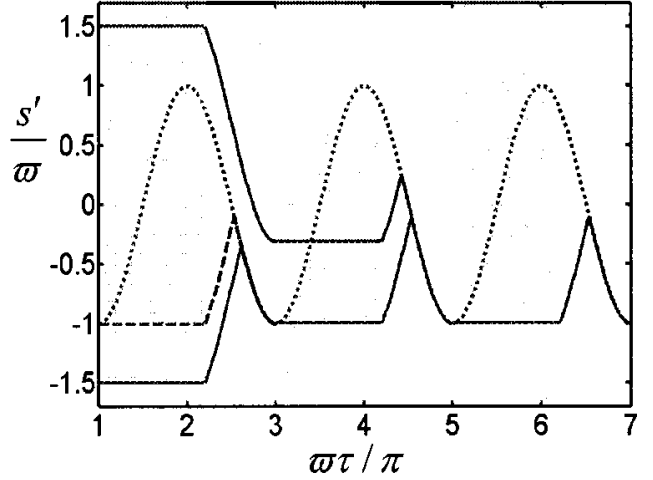

FIG. 8. Slider driven into periodic state. Shadowed regions indicate the noncontact phase. Two different initial slider velocities are considered, $s^{\prime}$ $=-1.5 \varpi$ and $s^{\prime}=1.5 \varpi$ at $\tau=\pi / \varpi$. Slider velocity as a function of time is plotted as solid lines. Dotted line is the horizontal velocity of the impact head. Dashed line is the periodic solution. $\beta \kappa / \varpi^{2}=1$ has been used in this simulation.

occurs at $\varpi \tau / \pi>2$. Note that contact cannot occur any earlier, i.e., $1<\varpi \tau / \pi<2$ since the vertical spring will be in tension. Since the slider velocity is greater than the velocity of the impact head (reverse slip), friction force will lower the slider velocity until the next separation, which must occur at $\sin \varpi \tau=0$. If the slider velocity is still higher than the impact head during the next contact, then friction force will again lower its velocity. This process continues until the slider velocity is smaller than the impact head (see Fig. 8, where one cycle is needed to satisfy this condition). Once the slider is slower than the impact head, slip will occur in the next contact. That is, the friction force will increase the slider velocity until it reaches the velocity of the impact head. Once this condition is met, stick occurs and periodic motion will occur after separation as shown earlier. For the second scenario, the slider velocity is smaller than the impact head at $\varpi \tau / \pi=1$. The friction force increases the slider velocity until stick occurs after which the slider motion will be periodic. Therefore, one expects that all initial conditions will lead to periodic motion, which is consistent with our simulation results. This fact allows us to show that only pull mode can occur in a system without horizontal impact compliance (assuming periodic motion has been achieved). Indeed, the slider moves with constant velocity $-\varpi$ during the noncontact phase and hence the displacement increment during this phase is $-\varpi \Delta \tau_{1}$, where $\Delta \tau_{1}$ is the duration of the noncontact phase and $\Delta \tau_{1} \geqslant \pi / \varpi$. In contact phase, the slider starts with velocity $-\varpi$ while the horizontal velocity of the impact head is somewhere between $[0, \varpi)$, depending on the position of the impact element. Thus slip will always occur in the beginning of an impact cycle and the friction force will always increase the slider velocity. As a result, the magnitude of the displacement increment of the slider during contact is always less than $\varpi \Delta \tau_{2}$, where $\Delta \tau_{2}$ is the contact duration which must be less than $\pi / \varpi$. Therefore, the displacement increment of a periodic slider motion $\Delta s_{p}$ is bounded by $\Delta s_{p}<-\varpi \Delta \tau_{1} \pm \varpi \Delta \tau_{2}<0$. To summarize, $\alpha=0$ always results in pull mode; thus, increasing $\alpha$ will favor the push mode. Furthermore, horizontal impact compliance is necessary for push mode to occur.

The separation between the regions of push and pull 

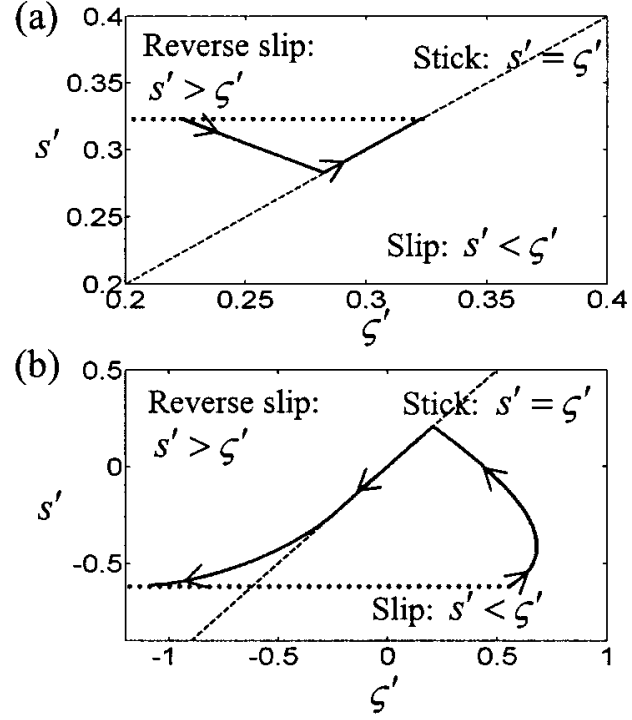

FIG. 9. Trajectories of the periodic solutions: (a) push mode, (b) pull mode. Solid lines are the trajectories in contact phase. Dotted lines are the trajectories in noncontact phase. Dashed lines denote the condition when the impact head and the slider stick together.

mode on the $\left(1 / \varpi^{2}, 1 / \alpha \kappa\right)$ plane is shown as dashed lines in Fig. 7. To find an equation of this separation criterion which applies to all combinations of $(\alpha, \beta, \kappa, \varpi)$, an equation of the form $\left(1 / \alpha \kappa-C_{1}\right) / \varpi^{2}=C_{2}$ is used to fit the integration results, where $C_{1}$ and $C_{2}$ are both functions of $\kappa$ and $\beta$. If $\left(1 / \alpha \kappa-C_{1}\right) / \varpi^{2}<C_{2}$, the slider is in push mode. When $\left(1 / \alpha \kappa-C_{1}\right) / \varpi^{2}>C_{2}$, the slider is in pull mode. This model satisfies the requirement that when $\alpha \rightarrow 0$, the slider is always in pull mode since $\varpi$ is bounded between $0.5 \leqslant \varpi$ $\leqslant 2$. Using a least-square fit, it is found that $C_{1} \approx[-\beta$ $+1 / \beta] /[22(\kappa+1)]$ and $C_{2}(\kappa, \beta) \approx 1 / \kappa$. Thus, the curve which separates push and pull mode is given by Eq. (9).

Equation (9) gives reasonable answers to three extreme cases, namely, $\kappa \rightarrow \infty$ (infinite friction), $\beta \rightarrow \infty$ (massless slider), and $\beta \rightarrow 0$ (massless impact head). When $\kappa \rightarrow \infty$, the separation curve approaches the two axes, $1 / \varpi^{2}=0$ and $1 / \alpha \kappa=0$, and the push mode disappears. This is because when impact friction is large, the slider and the impact head sticks to each other throughout contact. The slider has negative velocity when separation occurs; resulting in a negative displacement increment after one actuation cycle. For the case $\beta \rightarrow \infty$, i.e., $m \gg M$, push mode also disappears. In this case, the direction of motion of the slider follows the impact head. Because the slider and impact head is in contact during forward motion, the distance travelled by the slider during forward motion is less than the backward motion during the noncontact phase. Conversely, pull mode will vanish when $\beta \rightarrow 0$.

Two examples of trajectories of the periodic solutions in the velocity plane $\left(\zeta^{\prime}, s^{\prime}\right)$ are shown as solid lines in Fig. 9. Figure 9(a) shows a push mode trajectory with $\alpha=2.5$, $\varpi$ $=1.5, \beta=1.5$, and $\kappa=0.4$. When the slider separates from the impact head, it moves with constant velocity. Therefore, the trajectory in the noncontact phase is a horizontal line [dotted line in Fig. 8(a)]. Regions in the velocity plane where slip and reverse slip occur are separated by a dashed line, $\zeta^{\prime}$

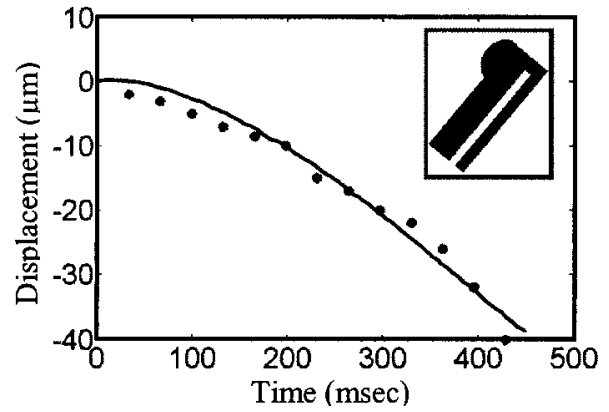

FIG. 10. Displacement of the slider with round impact head. Driving voltage is $8 \mathrm{~V}$. Dots are testing results. Solid line is integration results. The inset shows the shape of the impact head of the device under test.

$=s^{\prime}$, in Fig. 9(a). The trajectory shows that contact starts with reverse slip and that the slider and the impact head both have positive velocities. Friction force reduces the velocity of the slider and increase the impact head velocity until stick occurs. During contact, the velocity of the slider remains positive and results in a positive displacement increment of $\Delta s$ $=1.1796$. For the pull mode in Fig. 9(b), the parameters are $\alpha=0.5, \beta=1.5, \kappa=0.4$, and $\varpi=0.6$. The trajectory starts from slip and ends at reverse-slip contact with a negative velocity when the slider separated from the impact head, which causes negative displacement during the noncontact phase. The displacement increment of this periodic solution is $\Delta s=-3.5512$. These two examples indicate that the push/ pull mode is primarily controlled by the velocity of the slider at the instant of separation. This result is observed in all our numerical simulations.

\section{DEVICE CHARACTERIZATION AND MODEL VERIFICATION}

Electrothermal vibromotors with different configurations were fabricated using a three-layer polysilicon surface micromachining technology. Details can be found in Pai and Tien. ${ }^{10}$ The slider and the electrothermal actuators are realized from the second polysilicon layer which has thickness of $2 \mu \mathrm{m}$. The slider is composed of two beams which are $170 \mu \mathrm{m}$ long and exhibits a $40 \mu \mathrm{m}$ travel range. Beam width is varied from $5 \mu \mathrm{m}$ to $45 \mu \mathrm{m}$. The impact angle is fixed at $45^{\circ}$ for all vibromotor configurations. The shape of the impact head is round as shown in the inset of Fig. 10. The motion of the slider is recorded at 30 frames per second using a microscope. The electro thermal actuators are driven by ac waveform with zero dc bias. The input current has frequency ranges from $1 \mathrm{~Hz}$ to $15 \mathrm{kHz}$. It can be shown that the actuation frequency $\omega$ is twice the input current frequency if no dc bias has been applied. ${ }^{11}$

The displacement of the slider in pull mode $(5 \mu \mathrm{m}$ wide in each beam) is plotted against time in Fig. 10. The input current frequency is $500 \mathrm{~Hz}$. The measured displacement, denoted as dots in Fig. 10, is in good agreement with the prediction of our model with $\varpi=0.9, \alpha=0.1, \beta=0.9$, and $\kappa$ $=0.4$ with 30 impacts. The choice of 30 impacts is determined by these parameters. Specifically, the slider moves $40 \mu \mathrm{m}$ after 30 impacts with these parameters. Figure 10 shows that the slider has not reached its periodic state within 
(a)

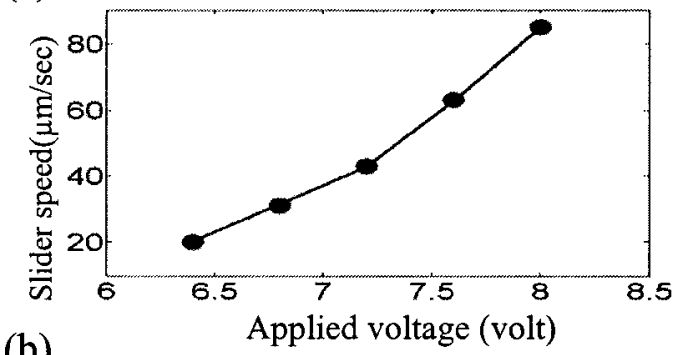

(b)

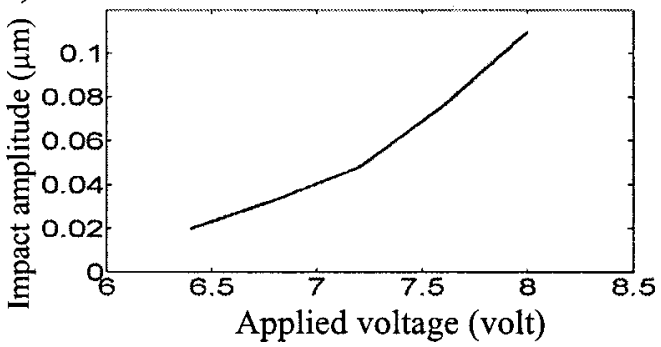

FIG. 11. (a) Slider speed vs applied voltage. Dots are testing results. Solid line is modeling results. (b) Impact amplitude used in the modeling.

30 impacts since the displacement increment is not linear with time. This is consistent with our numerical simulations which show that it takes at least 300 impacts before the slider can achieve periodic motion. It should also be noted that these fitting parameters may not be unique. Specifically, $\kappa$ $=0.4$ is chosen because the impact angle is $45^{\circ}$ and the coefficient of friction of polysilicon $\mu$ is taken to be $0.4 .{ }^{16}$ The impact amplitude is chosen to be $u=0.11 \mu \mathrm{m}$, which is about one-tenth of the initial gap between the impact head and the slider. This implies that the deflection of the actuator is about $1.11 \mu \mathrm{m}$, which is in the range of typical actuator deflections. The stiffness of the vertical spring is determined by choosing the resonance frequency of the impact head in the vertical direction to be $2 \pi \sqrt{k_{\nu} / m}=2.94 \mathrm{kHz}$, which is on the order of typical resonance frequencies of electrothermal actuators. Since the density of polysilicon is $\rho=2330 \mathrm{~kg} / \mathrm{m}^{3}$, the mass of half the slider can be calculated as $M=3.96$ $\times 10^{-3} \mu \mathrm{g}$ and that of the impact head has been assumed to be $m=0.036 \mu \mathrm{g}$.

Figure 10(a) shows that, for the same input current frequency of $500 \mathrm{~Hz}$, the average slider speed in the characterization [dots in Fig. 11(a)] increases with the applied input voltages. The average speed is defined as the total distance traveled by the slider divided by the total travel time. The total travel distance is fixed at $40 \mu \mathrm{m}$. According to the model in Eqs. (3a)-(3f) and its normalization, the actual displacement is directly proportional to the impact amplitude. When fitting the model into the characterization results with $\varpi=0.9, \alpha=0.1, \beta=9$, and $\kappa=0.4$, it is necessary to use different impact amplitude for different driving voltages. The impact amplitude used to fit the data in Fig. 11(a) is given in Fig. 11(b). As expected, increasing the applied voltage increases the amplitude of the impact element proportionally.

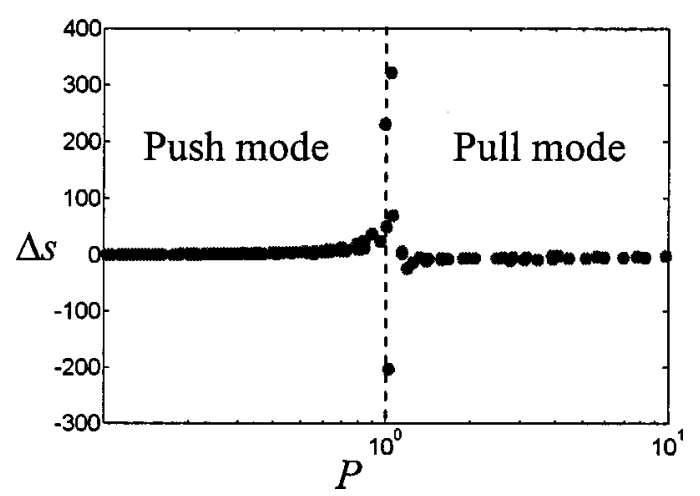

FIG. 12. Displacement increment $(\Delta s)$ as a function of $\lambda$ when $\kappa=0.4$ and $\beta=1.5$. The separation between push and pull modes is plotted by a dashed line, $\lambda=1$.

\section{DISCUSSION AND CONCLUSION}

Our results show that periodic solution with actuation frequency is possible if $0.5<\varpi<2$. In addition, the conditions for push/pull mode to occur in this regime $(0.5<\varpi<2)$ is

$$
\begin{aligned}
& \lambda<1 \text { (push mode). } \\
& \lambda>1 \text { (pull mode), }
\end{aligned}
$$

where $\lambda=\left(\kappa / \varpi^{2}\right)((1 / \alpha \kappa)-[(-\beta+1 / \beta) / 22(\kappa+1)])$. Figure 12 shows the displacement increment of one actuation cycle in periodic state as a function of $\lambda$ for $\kappa=0.4$ and $\beta=1.5$. It is found that the magnitude of the displacement increment increases as $\lambda$ approaches 1 . There is an abrupt velocity change at $\lambda=1$. As a result, although the slider moves with higher speed when $\lambda$ is closer to 1 , any fabrication variation could easily affect the property of contact and can cause an undesirable change of push/pull mode.

In conclusion, a continuous impact model based on Coulomb friction during the contact phase has been developed to simulate the dynamics of micromachined vibromotors. To simplify the model, the spring stiffness of the impact head is treated as phenomenological parameters. We showed that the horizontal impact compliance is necessary for the existence of push mode. Even with this simple model, the dynamics of the slider motion is extremely complicated. Our formulation shows that slider motion is controlled by four dimensionless parameters $\varpi, \alpha, \beta$, and $\kappa$. We demonstrated that there are regions in this parameter space where the motion of the impact head is periodic and has the same period as the thermal actuator. We also showed that there are regions in the parameter space where multiple impact as well as aperiodic solution can occur. Furthermore, we have also demonstrated the existence of pull and push mode as well as periodic solution and established a simple criterion for the achievement of these modes. In general, the pull mode can be suppressed by operation of the thermal actuator at a higher vibration frequency, i.e., increasing $\varpi$. Increasing $\alpha$ as well as decreasing $\beta$ and $\kappa$ also helps to suppress the pull mode. Finally, we fabricated and characterized a BETV to verify the ability of our model to predict pull mode. We hope our results can be used for the reliable operation of micromachined vibromotors. 


\section{ACKNOWLEDGMENT}

The authors would like to thank Professor Andy Ruina at Cornell University for his help in model development.

${ }^{1}$ N. C. Tien, O. Solgaard, M.-H. Kiang, M. Daneman, K. Y. Liu, and R. S. Muller, Sens. Actuators, A 52, 76 (1996).

${ }^{2}$ J. Liu, J. Y. Jeong, M. Pai, C. R. Pollock, N. C. Tien, and D. G. Ast, Proceedings of the IEEE/LEOS International Conference on Optical MEMS, Kaui, Hawaii (Aug. 21-24, 2000), 109.

${ }^{3}$ M. Pai and N. C. Tien, Proceedings of the 3rd International Conference on Micro Opto Electro Mechanical Systems, Mainz, Germany (Aug. 30-Sep. 1, 1999), 30.

${ }^{4}$ J. R. Reid, V. M. Bright, and J. T. Butler, Sens. Actuators, A 66, 292 (1998).

${ }^{5}$ K. Saitou, D.-A. Wang, and S. J. Wou, J. Microelectromech. Syst. 9, 336 (2000).

${ }^{6}$ J. H. Comtois and V. M. Bright, Sens. Actuators, A 58, 19 (1997)
${ }^{7}$ J. H. Comtois, M. A. Michalicek, and C. G. Barron, Sens. Actuators, A 70, 23 (1998).

${ }^{8}$ N. D. Mankame and G. K. Ananthasuresh, J. Micromech. Microeng. 11, 452 (2001).

${ }^{9}$ Q.-A. Huang and N. K. Lee, J. Micromech. Microeng. 9, 64 (1999).

${ }^{10} \mathrm{M}$. Pai and N. C. Tien, Proceedings of the 10th International Conference on Solid-State Sensors and Actuators (Transducers 1999), Sendai, Japan (June 7-10, 1999) Vol. 2, 1764.

${ }^{11}$ M. Pai, Development of a Surface Micromachined Electro-Thermal Vibromotor (Cornell University, Ithaca, NY, 2001).

${ }^{12}$ A. P. Lee, A. P. Pisano, and L. Lin, Proceedings of the IEEE Workshop on Micro Electro Mechanical Systems (MEMS 1991), Nara, Japan (Jan. 30-Feb. 2), 21.

${ }^{13}$ M. J. Daneman, N. C. Tien, O. Solgaard, A. P. Pisano, K. Y. Lau, and R. S. Muller, J. Microelectromech. Syst. 5, 159 (1996).

${ }^{14}$ G. Gilardi and I. Sharf, Mech. Mach. Theory 37, 1213 (2002)

${ }^{15}$ S. W. Shaw and P. Holmes, Phys. Rev. Lett. 51, 623 (1983).

${ }^{16}$ B. Bhushan and X. Li, J. Mater. Res. 12, 54 (1997). 